\section{Medical Care of the Pregnant Patient}

Karen Rosene-Montella, Erin Keely, Linda Anne Barbour and Richard V Lee

ISBN: 978-1-930513-86-0

Philadelphia: American College of Physicians, 2008

Pregnancy is a normal physiological process, usually well tolerated by most women. However, it predisposes at-risk mothers to pregnancy unique conditions. These mothers, along with pregnant women with chronic medical disorders, pose great challenges to their treating physicians. Concern regarding fetal welfare in addition to inadequate experience in dealing with emerging medical conditions during pregnancy may lead to suboptimal management. Furthermore, there is growing recognition of the impact of these medical complications to maternal mortality.

Medical Care of the Pregnant Patient aims to provide a framework for the care of pregnant patients with medical problems. The book has been written by multiple authors, who reflect the interdisciplinary nature of obstetric medicine. It is divided into 13 sections. The first two sections are introductory, discussing briefly preconception, genetic counselling and prescribing in pregnancy. This is followed by prenatal diagnosis, fetal surveillance and normal labour. From the obstetrician's point of view, these are dealt with superficially.
The chapter on diagnostic imaging is quite useful, benefiting from the addition of tables, which makes the information easy to assimilate. The section on behavioural and emotional health in pregnancy is interesting as these topics are usually omitted in similar texts. The remaining sections clearly outline the problems, current literature and management of almost all medical disorders encountered during pregnancy. The addition of new chapters, e.g. obesity, and separate ones covering pulmonary oedema, mechanical ventilation and vector-transmitted infections are welcome. However, the tables at the end of each chapter summarizing drug prescribing are confusing and repetitive. Future editions would benefit from more simple and concise organization, and possibly colour-coding of chapters.

Overall, I found this book to be clearly written, readable and fully referenced. It provides a welcome and ready reference to all those involved in the care of pregnant women, including obstetricians and internists, and would be a useful addition to every Obstetrics Department library.

\section{Naeema Mahmood}

Salmaniya Medical Complex, Consultant, Obstetrics and Gynaecology, PO Box 1164, Manama, Bahrain

Email: naeemamahmood@gmail.com

DOI: $10.1258 /$ om.2008.080021 